\title{
Favorable Shimada Classification
}

National Cancer Institute

\section{Source}

National Cancer Institute. Favorable Shimada Classification. NCI Thesaurus. Code C85414.

Patients of any age with stroma-rich tumors and no nodular pattern; or patients younger than 18 months with stroma-poor tumors, a mitosis-karyorrhexis index of less than 200, and differentiated or undifferentiated neuroblasts; or patients young er than 60 months with stroma-poor tumors, a mitosis-karyorrhexis index of less than 100 , and welldifferentiated neuroblasts. 